\title{
Deriving Non-determinism from Conjunction and Disjunction
}

\author{
Naijun Zhan ${ }^{1, \star}$ and Mila Majster-Cederbaum ${ }^{2}$ \\ 1 Lab. of Computer Science, Institute of Software, Chinese Academy of Sciences, \\ South Fourth Street No.4, Zhong Guan Cun, 100080, Beijing, P.R. China \\ 2 Lehrstuhl für Praktische Informatik II, Fakultät für Mathematik und Informatik, \\ Mannheim Universität, D7,27, 68163, Mannheim, Deutschland
}

\begin{abstract}
In this paper, we show that the non-deterministic choice "+", which was proposed as a primitive operator in Synchronization Tree Logic (STL for short) can be defined essentially by conjunction and disjunction in the $\mu$-calculus ( $\mu \mathrm{M}$ for short). This is obtained by extending the $\mu$ calculus with the non-deterministic choice "+" (denoted by $\mu \mathrm{M}^{+}$) and then showing that $\mu \mathrm{M}^{+}$can be translated into $\mu \mathrm{M}$. Furthermore, we also prove that STL can be encoded into $\mu \mathrm{M}^{+}$and therefore into $\mu \mathrm{M}$.
\end{abstract}

Keywords: Non-determinism, Synchronization Tree Logic, $\mu$-calculus, process algebra.

\section{Introduction}

Compositional methods allow one to build up a large system by composing existing systems with the defined constructors and reduce the problem of correctness for a complex system to similar and simpler correctness problems for the subsystems. Because the complexity of large systems is normally untractable, it is necessary that a method for developing these systems is compositional (vertically or horizontally) in order to avoid combinatorial explosion in specifying and verifying these systems.

It is widely agreed that modal and temporal logics such as the $\mu$-calculus [5] and Hennessy-Milner Logic (HML for short) [4], are an appropriate tool for the specification and proof of reactive systems. In many cases, these systems can be modelled by the term language $\mathcal{T}[\{\epsilon\},\{+\}$, Act, $\mathcal{X}]$ of an algebra with a congruence relation $\sim$, where $\mathcal{T}[\{\epsilon\},\{+\}, A c t, \mathcal{X}]$ is constructed from a constant $\epsilon$ by using a set Act of unary operators, a binary operator + and recursion. $\mathcal{T}[\{\epsilon\},\{+\}, A c t, \mathcal{X}]$ is at the base of many process algebras, where Act represents a set of action names, + the non-deterministic choice and $\epsilon$ the system performing no actions. The terms can be interpreted over trees labeled over Act synchronization trees - following the terminology of [8]. It is required that modal $\operatorname{logics} \mathcal{L}$ meet the condition of adequacy, namely,

$$
\forall t_{1}, t_{2} \in \mathcal{T}[\{\epsilon\},\{+\}, A c t, \mathcal{X}]\left(t_{1} \sim t_{2} \text { iff } \forall \phi \in \mathcal{L}\left(t_{1}=\phi \text { iff } t_{2}=\phi\right)\right) .
$$

\footnotetext{
* This work is supported in part by CNSF-60493200 and CNSF-60421001.
} 
I.e, the congruence $\sim$ and the equivalence relation induced by the logic agree. For example, HML has the property, i.e., two CCS terms are equal up to strong bisimulation if and only if they satisfy the same HML properties, see [4].

On the other hand, it is desirable that the logics have compositionality, i.e. there exists a connection between the connectives of these logics and the constructors of programs so that one can reduce the problem of correctness for a complex system to similar and simpler correctness problems for the subsystems. It seems that many classic modal logics like the $\mu$-calculus and HML do not have such a property.

Motivated by the above two requirements, Graf and Sifakis proposed a modal logic, called Synchronization Tree Logic (STL) [2]. The language of formulae of STL is generated from the constants $\epsilon, \top$ by using the boolean connectives, the set $2^{\text {Act }}$ of unary operators where Act is a set of actions, the binary operator + and fixpoint operators. The operator + of the logic is an extension of the one + of programs. $P=\phi_{1}+\phi_{2}$ means that there exist $P_{1}$ and $P_{2}$ such that $P \sim P_{1}+P_{2}$, $P_{1} \models \phi_{1}$ and $P_{2} \models \phi_{2}$. Therefore, $\mathcal{T}[\{\epsilon\},\{+\}, A c t, \mathcal{X}]$ is contained in STL, i.e., programs are formulae of the logic. In order to avoid confusions, we will use $\phi_{P}$ to denote the formula corresponding to the program $P$. So, the verification of an assertion $P \models \phi$ can be reduced to the syntax-directed proof of the validity of the formula $\phi_{P} \Rightarrow \phi$.

It is clear that STL is more expressive than $\mu \mathrm{M}$ since it is not hard to encode $\mu \mathrm{M}$ into STL, for example, $[A] \phi$ can be defined as $\neg\left(A \neg \phi_{\mathrm{STL}}+\top\right)$ and $\langle A\rangle \phi$ as $A \phi_{\mathrm{STL}}+\top$, where $A \subseteq A c t$ and $\phi_{\mathrm{STL}}$ stands for the counterpart of $\phi$ in STL. But for the converse direction, by our knowledge, it seems that until up to now it is still open.

In this paper, we will study the issue of the definability of + in $\mu \mathrm{M}$ and give an affirmative answer. We show that the choice + can be defined essentially by conjunction and disjunction in $\mu \mathrm{M}$. This is captured by extending $\mu \mathrm{M}$ with the choice + to $\mu \mathrm{M}^{+}$and then encoding $\mu \mathrm{M}^{+}$into $\mu \mathrm{M}$. Furthermore, we show that STL can be translated into $\mu \mathrm{M}^{+}$, and we can thus claim that $\mu \mathrm{M}$ is as expressive as STL.

The rest of this paper is organized as follows: Some basic notions are defined in Section 2. Section 3 briefly reviews $\mu \mathrm{M}$ firstly, then extends it with the nondeterministic choice + to $\mu \mathrm{M}^{+}$. Section 4 is devoted to encoding $\mu \mathrm{M}^{+}$into $\mu \mathrm{M}$. STL and some related results are provided in Section 5. Section 6 is devoted to translating STL into $\mu \mathrm{M}^{+}$. A short conclusion is given in Section 7 .

\section{Preliminaries}

Consider a term language $\mathcal{T}$ built from the constants $\epsilon, \tau$, and a set $\mathcal{X}$ of process variables by using a set Act of unary operators, a binary operator + , and recursion.

Formally, $\mathcal{T}$ is formed according to the following rules:

$$
\begin{aligned}
& -\epsilon, \tau \in \mathcal{T}, \mathcal{X} \subseteq \mathcal{T}, \\
& -a P, P_{1}+P_{2}, \text { rec } x . P \in \mathcal{T} \text { if } a \in \text { Act }, x \in \mathcal{X}, P, P_{1}, P_{2} \in \mathcal{T} .
\end{aligned}
$$


We denote by $\mathcal{T}[\{\epsilon\},\{+\}$, Act, $\mathcal{X}]$ the sub-language which consists of all the well-guarded and closed terms in $\mathcal{T}$, where rec x.P is well-guarded means that any occurrence of the variable $x$ in $P$ is within the scope of an operator of Act.

For a given $P \in \mathcal{T}$, the set of actions that occur in $P$ is called its sort, denoted $S(P)$, inductively defined by $S(\epsilon) \widehat{=} \emptyset, S(\tau) \widehat{=}$ Act, $S(x) \hat{=} \emptyset, S(a P) \widehat{=}\{a\} \cup$ $S(P), S\left(P_{1}+P_{2}\right) \hat{=} S\left(P_{1}\right) \cup S\left(P_{2}\right), S($ rec $x . P) \hat{=} S(P)$.

Intuitively, we consider that elements of $\mathcal{T}[\{\epsilon\},\{+\}, A c t, \mathcal{X}]$ represent programs: Act is a set of atomic actions; + stands for non-deterministic choice; and $\epsilon$ for the program performing no actions; $\tau$ can be conceived as a program that behaves like chaos in CSP [3] which can do anything.

A structured operational semantics of $\mathcal{T}$ in Plotkin's Style is defined as follows:

Act

$\overline{a P \stackrel{a}{\rightarrow} P}$

$\operatorname{Rec} \frac{P_{1}\left[\operatorname{rec} x \cdot P_{1} / x\right] \stackrel{a}{\rightarrow} P_{1}^{\prime}}{\operatorname{rec} x \cdot P_{1} \stackrel{a}{\rightarrow} P_{1}^{\prime}}$
$\mathrm{Nd}$

Chaos $\frac{a}{\tau \stackrel{a}{\rightarrow} Q}$ for any $a \in$ Act and $Q \in \mathcal{T}$.

A process term $P \in \mathcal{T}$ determines a labelled transition system, i.e., a tuple $\mathcal{T}(P)=(\Sigma, S(P), \rightarrow, P)$, where $\Sigma$ is the set of states which is reachable from $P$, and $P \in \Sigma$ is the initial state, $\rightarrow \subseteq \Sigma \times S(P) \times \Sigma$ is the set of transitions, derived from the above operational semantics.

Remark 1. 1. Any transition system representing a term of $\mathcal{T}[\{\epsilon\},\{+\}$, Act, $\mathcal{X}]$ is always finitely branching as only well-guarded terms are admitted;

2. The sort of each term of $\mathcal{T}[\{\epsilon\},\{+\}, A c t, \mathcal{X}]$ is finite as so is its syntax.

Definition 1. A binary relation $\mathcal{S}$ over $\mathcal{T}[\{\epsilon\},\{+\}$, Act, $\mathcal{X}]$ is called a strong bisimulation if $(P, Q) \in \mathcal{S}$ implies

- whenever $P \stackrel{a}{\rightarrow} P^{\prime}$ then, for some $Q^{\prime}, Q \stackrel{a}{\rightarrow} Q^{\prime}$ and $\left(P^{\prime}, Q^{\prime}\right) \in \mathcal{S}$, for any $a \in$ Act; and

- whenever $Q \stackrel{a}{\rightarrow} Q^{\prime}$ then, for some $P^{\prime}, P \stackrel{a}{\rightarrow} P^{\prime}$ and $\left(P^{\prime}, Q^{\prime}\right) \in \mathcal{S}$ for any $a \in$ Act.

Given two processes $P, Q \in \mathcal{T}[\{\epsilon\},\{+\}$, Act, $\mathcal{X}], P$ and $Q$ are strongly bisimilar, written $P \sim Q$, if $(P, Q) \in \mathcal{S}$ for some strong bisimulation $\mathcal{S}$.

It is shown in [7] that $\sim$ is a congruence on $\mathcal{T}[\{\epsilon\},\{+\}, A c t, \mathcal{X}]$. [1] proved the following result, namely,

Lemma 1. For each $P \in \mathcal{T}[\{\epsilon\},\{+\}$, Act, $\mathcal{X}]$, there exists a process of the form $\sum_{i=1}^{m} \Sigma_{j=1}^{i_{a_{i}}} a_{i} P_{i, j}$ such that $P \sim \Sigma_{i=1}^{m} \Sigma_{j=1}^{i_{a_{i}}} a_{i} P_{i, j}$, where $a_{i} \neq a_{j}$ if $i \neq j$.

Note that an empty sum is abbreviated as $\epsilon$.

\section{The $\mu$-Calculus and Its Extension with "+"}

In this section, we first briefly review the $\mu$-calculus; then extend the logic with the non-deterministic operator "+". We denote by $\mu \mathrm{M}^{+}$the extension. 
For easing to encode STL into $\mu \mathrm{M}$, we use the slightly generalized version of the $\mu$-calculus (see [9]) in the sense that modalities on sets of actions are adopted rather than modalities on a single action, although the two formalisms are equivalent if the set of actions is assumed to be finite.

\section{$3.1 \mu \mathrm{M}$}

Let $A c t$ be a set of atomic actions, ranged over by $a, b, c, \ldots A, B, \ldots$ stand for the subsets of Act. Let $t t$ be propositional constant as usual, and $\mathcal{X}$ be a set of variables, ranged over by $x, y, z, \ldots$.

Formulae of $\mu \mathrm{M}$ are generated by:

$$
\phi::=t t|x| \neg \phi|\phi \vee \phi|\langle A\rangle \phi|[A] \phi| \mu x . \phi,
$$

where $A \subseteq$ Act and $x \in \mathcal{X}$.

The notions of scope, bound and free occurrences of variables, closed and open formulae, etc. are the same as in first-order predicate logic, where $\mu x$ is treated as quantifier. We will use $f n(\phi)$ to stand for the variables that have some free occurrence in $\phi$, and $b n(\phi)$ for the variables that have some bound occurrence in $\phi$. We say that $\phi$ is positive (negative) in the variable $x$ if every free occurrence of $x$ in $\phi$ occurs within the scope of an even (odd) number of negations $\neg$. A formula $\phi$ is said positive (negative) if for every $x \in b n(\phi)$, its scope in $\phi$ is positive (negative) in $x$. A formula $\phi$ is called strongly positive if it is positive and each occurrence of $x$ is within the scope of an even number of negations $\neg$ for any $x \in f n(\phi)$. For example, let $\phi_{1} \widehat{=} x \vee \mu x . \neg \neg x, \phi_{2} \widehat{=} \neg y \vee \mu x . \neg \neg x$. It is clear that $\phi_{1}$ and $\phi_{2}$ both are positive; however, $\phi_{1}$ is strongly positive as well, but $\phi_{2}$ is not. We say that $x$ is guarded in $\phi$ if every occurrence of $x$ in $\phi$ is within the scope of $\langle A\rangle$ or $[A]$ for some $A \subseteq$ Act. A formula $\phi$ is called guarded if each variable in $b n(\phi)$ is guarded.

If $A=\{a\}$, we directly write $\langle a\rangle \phi$ and $[a] \phi$ instead of $\langle\{a\}\rangle \phi$ and $[\{a\}] \phi$ respectively.

We denote by $\mathcal{L}_{\mu}($ Act $)$ the language of formulae of $\mu \mathrm{M}$ that are positive and guarded, by $c \mathcal{L}_{\mu}(A c t)$ the set of all closed formulae in $\mathcal{L}_{\mu}(A c t)$. As [11] showed that any formula $\phi \in \mu \mathrm{M}$ is equivalent to a positive guarded formula $\phi^{\prime}$, we theorefore only focus on $\mathcal{L}_{\mu}(A c t)$ and $c \mathcal{L}_{\mu}(A c t)$ in what follows.

A valuation $\rho$ is a mapping with the type $\rho: \mathcal{X} \rightarrow 2^{\mathcal{T}[\{\epsilon\},\{+\}, A c t, \mathcal{X}]}$, which associates a set of processes with each propositional variable. $\rho[x \sim \mathcal{A}]$ agrees with $\rho$ except for assigning $\mathcal{A}$ to $x$.

Definition 2. The semantics of $\mathcal{L}_{\mu}($ Act $)$ under a valuation $\rho$ is given by a satisfaction relation between $\mathcal{T}[\{\epsilon\},\{+\}$, Act, $\mathcal{X}]$ and $\mathcal{L}_{\mu}($ Act $)$ relative to $\rho$, denoted by $\models_{\mu \mathrm{M}}^{\rho}$, inductively defined as follows: 


$$
\begin{gathered}
P \models{ }_{\mu \mathrm{M}}^{\rho} t t, \\
P \models{ }_{\mu \mathrm{M}}^{\rho} x, \text { iff } P \in \rho(x), \\
P \models_{\mu \mathrm{M}}^{\rho} \neg \phi \text { iff } P \models_{\mu \mathrm{M}}^{\rho} \phi, \\
P \models{ }_{\mu \mathrm{M}}^{\rho} \phi_{1} \vee \phi_{2} \text { iff } P=_{\mu \mathrm{M}}^{\rho} \phi_{1} \text { or } P \models{ }_{\mu \mathrm{M}}^{\rho} \phi_{2}, \\
P \models{ }_{\mu \mathrm{M}}^{\rho}\langle A\rangle \phi \text { iff } \exists a \in A, \exists P^{\prime} . P \stackrel{a}{\rightarrow} P^{\prime} \text { and } P^{\prime} \models_{\mu \mathrm{M}}^{\rho} \phi, \\
P \models{ }_{\mu \mathrm{M}}^{\rho}[A] \phi \text { iff } \forall a \in A, \forall P^{\prime} . P \stackrel{a}{\rightarrow} P^{\prime} \text { implies } P^{\prime} \models_{\mu \mathrm{M}}^{\rho} \phi, \\
P \models{ }_{\mu \mathrm{M}}^{\rho} \mu x . \phi \text { iff } P \in \bigcap\left\{\mathcal{A} \mid\left\{Q \mid Q \models_{\mu \mathrm{M}}^{\rho[x \sim \mathcal{A}]} \phi\right\} \subseteq \mathcal{A}\right\},
\end{gathered}
$$

where $P, P^{\prime} \in \mathcal{T}[\{\epsilon\},\{+\}$, Act, $\mathcal{X}]$ and $\mathcal{A} \subseteq \mathcal{T}[\{\epsilon\},\{+\}$, Act, $\mathcal{X}]$.

Note that the restriction that all formulae of $\mathcal{L}_{\mu}($ Act $)$ are positive guarantees that the interpretation of a formula of the form $\mu x . \phi$ is well defined by the Tarski-Knaster Theorem [10].

Since the meaning of a closed formula $\phi$ is independent of valuations, we will abbreviate $P=_{\mu \mathrm{M}}^{\rho} \phi$ as $P \models{ }_{\mu \mathrm{M}} \phi$ for any valuation $\rho$.

The following derived operators are useful:

$$
\begin{aligned}
f f & \hat{=} \neg t t, \\
\phi_{1} \wedge \phi_{2} & \widehat{=} \neg\left(\left(\neg \phi_{1}\right) \vee\left(\neg \phi_{2}\right)\right), \\
\phi_{1} \Rightarrow \phi_{2} & \hat{=}\left(\neg \phi_{1}\right) \vee \phi_{2}, \\
\phi_{1} \Leftrightarrow \phi_{2} & \widehat{=}\left(\phi_{1} \Rightarrow \phi_{2}\right) \wedge\left(\phi_{2} \Rightarrow \phi_{1}\right), \\
\nu x . \phi & \widehat{=} \neg(\mu x . \neg \phi\{\neg x / x\}) .
\end{aligned}
$$

Convention: In order to improve the readability, in the later, we assume the

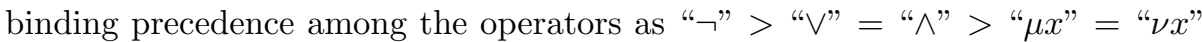
$>" \Rightarrow "=" \Leftrightarrow "$

\section{$3.2 \mu \mathrm{M}^{+}$}

$\mu \mathrm{M}^{+}$is an extension of $\mu \mathrm{M}$ with the non-deterministic choice "+". Informally, $\phi+\psi$ holds in a process $P$ means that there exist $P_{1}$ and $P_{2}$ such that $P \sim P_{1}+P_{2}$, $P_{1}$ satisfies $\phi$ and $P_{2}$ meets $\psi$.

Given a set $A c t$ of atomic actions and a set $\mathcal{X}$ of variables, formulae of $\mu \mathrm{M}^{+}$ are generated as follows:

$$
\phi::=t t|x| \neg \phi|\phi \vee \phi|\langle A\rangle \phi|[A] \phi| \phi+\phi \mid \mu x . \phi,
$$

where $x \in \mathcal{X}$ and $A \subseteq A c t$.

Some notions for $\mu \mathrm{M}^{+}$can be defined same as in $\mu \mathrm{M}$. We will use $\mathcal{L}_{\mu}^{+}($Act $)$ to denote the language of formulae of $\mu \mathrm{M}^{+}$that are guarded and positive and $c \mathcal{L}_{\mu}^{+}(A c t)$ to stand for the set of closed formulae in $\mathcal{L}_{\mu}^{+}(A c t)$ 
Definition 3. A formula $\phi \in \mathcal{L}_{\mu}^{+}($Act $)$is called strictly guarded, if each variable $x \in f n(\phi) \cup b n(\phi)$ is guarded and does not occur in any sub-formula of the forms $x+\psi$ or $\neg x+\psi$.

Note that strictly guarded is stronger than guarded, for instance, $\langle A\rangle(x+y)$ is guarded, but not strictly guarded.

Definition 4. The semantics of $\mathcal{L}_{\mu}^{+}($Act $)$under a given valuation $\rho$ is given by a satisfaction relation between $\mathcal{T}[\{\epsilon\},\{+\}$, Act, $\mathcal{X}]$ and $\mathcal{L}_{\mu}^{+}($Act $)$relative to $\rho$, denoted by $\models_{\mu \mathrm{M}^{+}}^{\rho}$. The definition of $\models_{\mu \mathrm{M}^{+}}^{\rho}$ contains all clauses listed in Definition 2, in addition to including the following clause for interpreting "+":

$$
P \models{ }_{\mu \mathrm{M}^{+}}^{\rho} \phi_{1}+\phi_{2} \text { iff } \exists P_{1} \exists P_{2} . P \sim P_{1}+P_{2}, P_{1} \models_{\mu \mathrm{M}^{+}}^{\rho} \phi_{1} \text { and } P_{2} \models_{\mu \mathrm{M}^{+}}^{\rho} \phi_{2},
$$

where $P, P_{1}, P_{2} \in \mathcal{T}[\{\epsilon\},\{+\}$, Act, $\mathcal{X}]$.

Since the meaning of a closed formula $\phi$ is independent of valuations, we will abbreviate $P \models_{\mu \mathrm{M}^{+}}^{\rho} \phi$ as $P \models_{\mu \mathrm{M}^{+}} \phi$ for any valuation $\rho$. A formula $\phi$ is valid, written $\models_{\mu \mathrm{M}^{+}} \phi$, if $P \models_{\mu \mathrm{M}^{+}}^{\rho} \phi$ for any $P \in \mathcal{T}[\{\epsilon\},\{+\}$, Act, $\mathcal{X}]$ and any valuation $\rho$. Sometimes, we write $\phi$ directly instead of $\models_{\mu \mathrm{M}^{+}} \phi$ for simplicity.

It is clear that $\mathcal{L}_{\mu}(A c t) \subseteq \mathcal{L}_{\mu}^{+}(A c t)$ and $c \mathcal{L}_{\mu}(A c t) \subseteq c \mathcal{L}_{\mu}^{+}(A c t)$. Convention We will assume that "+" has a priority over all other binary operators, but " $\neg$ " has a higher priority to it. Given a set $A \subset B$, we will use $\bar{A}$ to stand for the complement $B-A$.

\subsection{Some Results on $\mu \mathrm{M}$ and $\mu \mathrm{M}^{+}$}

From Definition 4, it is easy to see that "+" is monotonic. That is,

Proposition 1. If $\phi_{1} \Rightarrow \phi_{2}$ and $\psi_{1} \Rightarrow \psi_{2}$ then $\phi_{1}+\psi_{1} \Rightarrow \phi_{2}+\psi_{2}$.

Definition 5. Given a set of process $\mathcal{A} \subseteq \mathcal{T}[\{\epsilon\},\{+\}$, Act, $\mathcal{X}], \mathcal{A}$ is bisimulation closed if $\forall P \in \mathcal{A}$ and $\forall Q \in \mathcal{T}[\{\epsilon\},\{+\}$, Act, $\mathcal{X}], P \sim Q$ implies that $Q \in \mathcal{A}$. For convenience, from now on, we will abbreviate bisimulation closed as B.C.. $A$ valuation $\rho$ is B.C. if for all $x \in \mathcal{X} \rho(x)$ is B.C..

Regarding the above definition, we have the following results:

Lemma 2. If $\mathcal{A}_{1}, \mathcal{A}_{2} \subseteq \mathcal{T}[\{\epsilon\},\{+\}$, Act, $\mathcal{X}]$ are B.C., then

1. $\overline{\mathcal{A}}_{1}, \mathcal{A}_{1} \cap \mathcal{A}_{2}$ and $\mathcal{A}_{1} \cup \mathcal{A}_{2}$ are B.C.,

2. $\left\{P \in \mathcal{T}[\{\epsilon\},\{+\}\right.$, Act, $\mathcal{X}] \mid$ if $P \stackrel{a}{\rightarrow} P^{\prime}$ and $a \in A$ then $\left.P^{\prime} \in \mathcal{A}_{1}\right\}$ is B.C.,

3. $\left\{P \in \mathcal{T}[\{\epsilon\},\{+\}, A c t, \mathcal{X}] \mid \exists P^{\prime} \in \mathcal{A}_{1} . \exists a \in A . P \stackrel{a}{\rightarrow} P^{\prime}\right\}$ is B.C.,

4. $\mathcal{A}_{1}+\mathcal{A}_{2}$ is B.C., where $\mathcal{A}_{1}+\mathcal{A}_{2}$ denotes the set $\left\{P \mid \exists P_{1} \in \mathcal{A}_{1} \cdot \exists P_{2} \in \mathcal{A}_{2} . P \sim\right.$ $\left.P_{1}+P_{2}\right\}$.

For any set of processes $\mathcal{A} \subseteq \mathcal{T}[\{\epsilon\},\{+\}, A c t, \mathcal{X}]$, we can associate with it the following subset:

$$
\mathcal{A}^{d} \widehat{=}\{P \in \mathcal{A} \mid \text { if } P \sim Q \text { and } Q \in \mathcal{T}[\{\epsilon\},\{+\}, \text { Act, } \mathcal{X}] \text { then } Q \in \mathcal{A}\} .
$$

The set $\mathcal{A}^{d}$ is the largest bisimulation closed set contained in $\mathcal{A}$. 
Lemma 3. For any set $\mathcal{A}, \mathcal{A}_{i} \subseteq \mathcal{T}[\{\epsilon\},\{+\}$, Act, $\mathcal{X}]$ for $i=1,2$,
1. $\mathcal{A}^{d}$ is B.C.,
2. $\mathcal{A}^{d} \subseteq \mathcal{A}$,
3. $\mathcal{A}^{d}=\mathcal{A}$ if $\mathcal{A}$ is B.C.,
4. $\mathcal{A}_{1}^{d} \subseteq \mathcal{A}_{2}^{d}$ if $\mathcal{A}_{1} \subseteq \mathcal{A}_{2}$,
5. $\mathcal{A}_{1}^{d}+\mathcal{A}_{2}^{d} \subseteq \mathcal{A}^{d}$ if $\mathcal{A}_{1}+\mathcal{A}_{2} \subseteq \mathcal{A}$.

We use $\rho^{d}$ to stand for the valuation defined by $\rho^{d}(x)=\rho(x)^{d}$. By Lemma 3, it is clear that $\rho^{d}$ is B.C. for any valuation $\rho$. From now on, we will use $B C V$ to stand for the set of bisimulation closed valuations.

In the following, we will use $\|\phi\|_{\rho}$ to denote the set of processes that meet $\phi$ under the valuation $\rho$, i.e., $\|\phi\|_{\rho} \widehat{=}\left\{P \in \mathcal{T}[\{\epsilon\},\{+\}\right.$, Act, $\left.\mathcal{X}] \mid P \models_{\mu \mathrm{M}^{+}}^{\rho} \phi\right\}$. We will write $\rho \subseteq \rho^{\prime}$ if $\rho(x) \subseteq \rho^{\prime}(x)$ for any $x \in \mathcal{X}$.

Proposition 2. For any $\phi \in \mathcal{L}_{\mu}^{+}($Act $)$, if $\phi$ is strongly positive and $\rho \subseteq \rho^{\prime}$, then $\|\phi\|_{\rho} \subseteq\|\phi\|_{\rho^{\prime}}$.

Lemma 4. For any $\phi \in \mathcal{L}_{\mu}^{+}($Act $)$which is strongly positive, any valuation $\rho$, and $\mathcal{A} \in \mathcal{T}[\{\epsilon\},\{+\}$, Act, $\mathcal{X}]$, then

1. If $\rho$ is B.C., then $\|\phi\|_{\rho}$ is B.C. as well;

2. $\|\phi\|_{\rho^{d}} \subseteq \mathcal{A}^{d}$ if $\|\phi\|_{\rho} \subseteq \mathcal{A}$.

Proof. Similar to the proof for Proposition 3 in Section 5.4 in [9], simultaneously proving these two statements by induction on $\phi$, the proof is done.

As [9] pointed out that each formula of $c \mathcal{L}_{\mu}(A c t)$ defines a bisimulation invariant property, the following theorem indicates that every formula in $c \mathcal{L}_{\mu}^{+}(A c t)$ is bisimulation invariant as well. The forward direction of the theorem follows immediately from the above lemma; the converse direction comes from the fact $c \mathcal{L}_{\mu}(A c t) \subseteq c \mathcal{L}_{\mu}^{+}(A c t)$.

Theorem 1. For any $P, Q \in \mathcal{T}[\{\epsilon\},\{+\}$, Act, $\mathcal{X}], P \sim Q$ iff for each $\phi \in$ $c \mathcal{L}_{\mu}^{+}($Act $), P=_{\mu \mathrm{M}^{+}} \phi$ iff $Q \models_{\mu \mathrm{M}^{+}} \phi$.

The following lemmas can be proved by Definition 4 .

\section{Lemma 5.}

(1) $\phi+f f \Leftrightarrow f f$

(3) $[A] t t \Leftrightarrow t t$

(5) $\phi+\psi \Leftrightarrow \psi+\phi$

(7) $\langle A\rangle \phi_{1} \wedge[A \cup B] \phi_{2} \Rightarrow\langle A\rangle\left(\phi_{1} \wedge \phi_{2}\right)$

(9) $\langle A\rangle \phi_{1} \vee\langle A\rangle \phi_{2} \Leftrightarrow\langle A\rangle\left(\phi_{1} \vee \phi_{2}\right)$

(11) $\left\langle A_{1} \cup A_{2}\right\rangle \phi \Leftrightarrow\left\langle A_{1}\right\rangle \phi \vee\left\langle A_{2}\right\rangle \phi$
(2) $t t+t t \Leftrightarrow t t$

(4) $\langle A\rangle f f \Leftrightarrow f f$

(6) $(\phi+\psi)+\varphi \Leftrightarrow \phi+(\psi+\varphi)$

(8) $\phi+(\varphi \vee \psi) \Leftrightarrow(\phi+\varphi) \vee(\phi+\psi)$

(10) $[A] \phi_{1} \wedge[A] \phi_{2} \Leftrightarrow[A]\left(\phi_{1} \wedge \phi_{2}\right)$

(12) $\left[A_{1} \cup A_{2}\right] \phi \Leftrightarrow\left[A_{1}\right] \phi \wedge\left[A_{2}\right] \phi$

\section{Lemma 6.}

(1) $\neg[A] \phi \Leftrightarrow\langle A\rangle \neg \phi$

(2) $\neg\langle A\rangle \phi \Leftrightarrow[A] \neg \phi$

(3) $\left[A_{1}\right] \phi_{1} \wedge\left[A_{2}\right] \phi_{2} \Leftrightarrow\left[A_{1}-\left(A_{1} \cap A_{2}\right)\right] \phi_{1} \wedge\left[A_{1} \cap A_{2}\right]\left(\phi_{1} \wedge \phi_{2}\right) \wedge$ $\left[A_{2}-\left(A_{1} \cap A_{2}\right)\right] \phi_{2}$ 


\section{Reducing $c \mathcal{L}_{\mu}^{+}($Act $)$to $c \mathcal{L}_{\mu}($ Act $)$}

In this section, we show that "+" is definable in $\mu \mathrm{M}$ by reducing $c \mathcal{L}_{\mu}^{+}($Act $)$ into $c \mathcal{L}_{\mu}(A c t)$. The encoding is completed via the following three steps: firstly, we prove that in some special cases, "+" can be defined by conjunction and disjunction; then we show that the problem of eliminating "+" in a strongly positive and strictly guarded formula $\phi$ can be reduced to one of the above special cases; and finally we complete the encoding by proving that for any $\phi \in c \mathcal{L}_{\mu}^{+}(A c t)$, there is a formula $\phi^{\prime} \in c \mathcal{L}_{\mu}^{+}(A c t)$ which is strictly guarded such that $\phi \Leftrightarrow \phi^{\prime}$.

We say that $\phi$ implies $\psi$ w.r.t. bisimulation closed valuations, denoted by $\phi \stackrel{b c}{\Rightarrow} \psi$, if $\|\phi\|_{\rho} \subseteq\|\psi\|_{\rho}$ for any $\rho \in B C V . \phi \stackrel{b c}{\Leftrightarrow} \psi$ means $\phi \stackrel{b c}{\Rightarrow} \psi$ and $\psi \stackrel{b c}{\Rightarrow} \phi$. It is clear that $\phi \Rightarrow \psi$ implies $\phi \stackrel{b c}{\Rightarrow} \psi$, and $\phi \stackrel{b c}{\Rightarrow} \psi$ iff $\phi \Rightarrow \psi$ if $\phi, \psi \in c \mathcal{L}_{\mu}^{+}($Act $)$.

In order to attain the first step, we need the following proposition:

Proposition 3. 1. For any $P, Q \in \mathcal{T}[\{\epsilon\},\{+\}$, Act, $\mathcal{X}]$, if $P \models_{\mu \mathrm{M}^{+}}^{\rho}\langle A\rangle \phi$ then $P+Q \models{ }_{\mu \mathrm{M}^{+}}^{\rho}\langle A\rangle \phi ;$ and

2. If $P \models_{\mu \mathrm{M}^{+}}^{\rho}[A] \phi_{1}$ and $Q \models_{\mu \mathrm{M}^{+}}^{\rho}[A] \phi_{2}$ then $P+Q \models_{\mu \mathrm{M}^{+}}^{\rho}[A]\left(\phi_{1} \vee \phi_{2}\right)$.

The following lemma claims that in some special cases, "+" can be defined essentially by conjunction and disjunction.

\section{Lemma 7.}

$$
\begin{aligned}
&\left.\bigwedge_{i=1}^{n} \bigwedge_{j=1}^{n_{i}}\left\langle A_{i}\right\rangle \phi_{i, j} \wedge \bigwedge_{i=1}^{m}\left[B_{i}\right] \psi_{i}\right)+\left(\bigwedge_{i=1}^{k} \bigwedge_{j=1}^{k_{i}}\left\langle C_{i}\right\rangle \varphi_{i, j} \wedge \bigwedge_{i=1}^{m}\left[B_{i}\right] \chi_{i}\right) \\
& \stackrel{b c}{\Leftrightarrow} \bigwedge_{i=1}^{n} \bigwedge_{j=1}^{n_{i}}\left\langle A_{i}\right\rangle\left(\phi_{i, j} \wedge \psi_{i}\right) \wedge \bigwedge_{i=1}^{k} \bigwedge_{j=1}^{k_{i}}\left\langle C_{i}\right\rangle\left(\varphi_{i, j} \wedge \chi_{i}\right) \wedge \bigwedge_{i=1}^{m}\left[B_{i}\right]\left(\psi_{i} \vee \chi_{i}\right)
\end{aligned}
$$

where all conjuncts in the formula of the left side of $\stackrel{b c}{\Leftrightarrow}$ are strongly positive, $n, k \leq m, \forall 1 \leq i \leq n . A_{i} \subseteq B_{i}, \forall 1 \leq i \leq k . C_{i} \subseteq B_{i}$, and for any $1 \leq i, j \leq m$, if $i \neq j$ then $B_{i} \cap B_{j}=\emptyset$.

Proof. " $\stackrel{b c}{\Rightarrow}$ " can be easily proved by Proposition 3 and Lemma 4 . So, we only give a sketch for the proof of the converse direction. Assume

$$
P=_{\mu \mathrm{M}^{+}}^{\rho} \bigwedge_{i=1}^{n} \bigwedge_{j=1}^{n_{i}}\left\langle A_{i}\right\rangle\left(\phi_{i, j} \wedge \psi_{i}\right) \wedge \bigwedge_{i=1}^{k} \bigwedge_{j=1}^{k_{i}}\left\langle C_{i}\right\rangle\left(\varphi_{i, j} \wedge \chi_{i}\right) \wedge \bigwedge_{i=1}^{m}\left[B_{i}\right]\left(\psi_{i} \vee \chi_{i}\right)
$$

where $\rho$ is B.C.. By Lemma $1, P \sim \Sigma_{i=1}^{l} \Sigma_{j=1}^{i_{a_{i}}} a_{i} P_{i, j}$, where $l \geq m$ and for any $1 \leq i, j \leq l$, if $i \neq j$ then $a_{i} \neq a_{j}$. So, we have $\Sigma_{i=1}^{l} \Sigma_{j=1}^{i_{a_{i}}} a_{i} P_{i, j} \models_{\mu \mathrm{M}^{+}}^{\rho}$ $\bigwedge_{i=1}^{n} \bigwedge_{j=1}^{n_{i}}\left\langle A_{i}\right\rangle\left(\phi_{i, j} \wedge \psi_{i}\right)$ by Lemma 4 . This implies that for each $1 \leq i \leq n$ and 
$1 \leq j \leq n_{i}$, there exist $1 \leq r_{i} \leq l$ and $1 \leq h_{j} \leq i_{a_{r_{i}}}$ such that $a_{r_{i}} \in A_{i}$ and $P_{r_{i}, h_{j}} \models_{\mu \mathrm{M}+}^{\rho} \phi_{i, j} \wedge \psi_{i}$. Let $P^{\prime} \widehat{=} \sum_{i=1}^{n} \Sigma_{j=1}^{n_{i}} a_{r_{i}} P_{r_{i}, h_{j}}$. It is obvious that

$$
P^{\prime} \models_{\mu \mathrm{M}+}^{\rho} \bigwedge_{i=1}^{n} \bigwedge_{j=1}^{n_{i}}\left\langle A_{i}\right\rangle \phi_{i, j} \wedge \bigwedge_{i=1}^{m}\left[B_{i}\right] \psi_{i}
$$

Similarly, we get that for each $1 \leq i \leq k$ and $1 \leq j \leq k_{i}$, there exist $1 \leq$ $r_{i} \leq l$ and $1 \leq h_{j} \leq i_{a_{r_{i}}}$ such that $a_{r_{i}} \in C_{i}$ and $P_{r_{i}, h_{j}} \models_{\mu \mathrm{M}^{+}}^{\rho} \varphi_{i, j} \wedge \chi_{i}$. Let $P^{\prime \prime} \widehat{=} \Sigma_{i=1}^{k} \Sigma_{j=1}^{n_{i}} a_{r_{i}} P_{r_{i}, h_{j}}$. It is easy to show that

$$
P^{\prime \prime} \models{ }_{\mu \mathrm{M}+}^{\rho} \bigwedge_{i=1}^{k} \bigwedge_{j=1}^{k_{i}}\left\langle C_{i}\right\rangle \varphi_{i, j} \wedge \bigwedge_{i=1}^{m}\left[B_{i}\right] \chi_{i}
$$

Then, we add each summand of $\Sigma_{i=1}^{l} \Sigma_{j=1}^{i_{a_{i}}} a_{i} P_{i, j}$ to $P^{\prime}$ or $P^{\prime \prime}$ according to the following algorithm: For each $1 \leq i \leq l$, if $a_{i} \in B_{j}$ for some $j \in\{1, \ldots, m\}$ then let $I_{1} \widehat{=}\left\{h \mid P_{i, h} \models \psi_{j}\right\}$ and $I_{2} \widehat{=}\left\{h \mid P_{i, h} \models \chi_{j}\right\}$; otherwise, $I_{1} \widehat{=}\left\{1, \ldots, i_{a_{i}}\right\}$ and $I_{2}=\emptyset$. Since $P \models_{\mu \mathrm{M}^{+}}^{\rho}\left[B_{j}\right]\left(\psi_{j} \vee \chi_{j}\right)$, it is clear that $I_{1} \cup I_{2}=\left\{1, \ldots, i_{a_{i}}\right\}$. Then, let $P^{\prime}:=P^{\prime}+\sum_{h \in I_{1}} a_{i} P_{i, h}$ and $P^{\prime \prime}:=P^{\prime \prime}+\sum_{h \in I_{2}} a_{i} P_{i, h}$. Because $B_{i} \cap B_{j}=\emptyset$ if $i \neq j$, it is easy to show that (2) and (3) keep invariant for each cojoining.

Additionally, it is easy to see that $P^{\prime}+P^{\prime \prime} \sim P$. Hence, from Lemma 4 ,

$$
P \mid=_{\mu \mathrm{M}^{+}}^{\rho}\left(\bigwedge_{i=1}^{n} \bigwedge_{j=1}^{n_{i}}\left\langle A_{i}\right\rangle \phi_{i, j} \wedge \bigwedge_{i=1}^{m}\left[B_{i}\right] \psi_{i}\right)+\left(\bigwedge_{i=1}^{k} \bigwedge_{j=1}^{k_{i}}\left\langle C_{i}\right\rangle \varphi_{i, j} \wedge \bigwedge_{i=1}^{m}\left[B_{i}\right] \chi_{i}\right)
$$

Furthermore, applying the above lemma, we can complete the second step by proving the following results:

Lemma 8. For any $\phi \in \mathcal{L}_{\mu}^{+}(A c t)$, if $\phi$ is strictly guarded and strongly positive, then there exists $\phi^{\prime}$ in which no + occurs such that $\phi^{\prime} \stackrel{b c}{\Leftrightarrow} \phi$ and $\phi^{\prime}$ is strictly guarded and strongly positive.

Proof. By induction on the structure of $\phi$. Here, we only list the proofs for some interesting cases.

. $\phi=\neg \psi$

Suppose $\rho \in B C V$ and $f n(\phi) \subseteq\left\{x_{1}, \ldots, x_{n}\right\}$. Let $\neg \rho$ be defined by $\neg \rho(x)=$ $\mathcal{T}[\{\epsilon\},\{+\}, A c t, \mathcal{X}]-\rho(x)$ for any $x \in \mathcal{X}$. By Lemma 2.1., $\neg \rho$ is B.C.. It is easy to see that $\|\varphi\|_{\rho}=\left\|\varphi\left\{\neg x_{1} / x_{1}, \ldots, \neg x_{n} / x_{n}\right\}\right\|_{\neg \rho}$ for any $\varphi \in \mathcal{L}_{\mu}^{+}($Act $)$ whose free variables are in $\left\{x_{1}, \ldots, x_{n}\right\}$.

Since $\phi$ is strictly guarded and strongly positive, so is $\psi\left\{\neg x_{1} / x_{1}, \ldots, \neg x_{n} / x_{n}\right\}$. By the induction hypothesis, there is $\psi^{\prime}$ in which no + occurs such that $\psi\left\{\neg x_{1} / x_{1}, \ldots, \neg x_{n} / x_{n}\right\} \stackrel{b c}{\Leftrightarrow} \psi^{\prime}$ and $\psi^{\prime}$ is strictly guarded and strongly posi- 
tive. Besides,

$$
\begin{aligned}
\|\phi\|_{\rho} & =\mathcal{T}[\{\epsilon\},\{+\}, A c t, \mathcal{X}]-\|\psi\|_{\rho} \\
& =\mathcal{T}[\{\epsilon\},\{+\}, A c t, \mathcal{X}]-\left\|\psi\left\{\neg x_{1} / x_{1}, \ldots, \neg x_{n} / x_{n}\right\}\right\|_{\neg \rho} \\
& =\mathcal{T}[\{\epsilon\},\{+\}, A c t, \mathcal{X}]-\left\|\psi^{\prime}\left\{\neg x_{1} / x_{1}, \ldots, \neg x_{n} / x_{n}\right\}\right\|_{\rho} \\
& =\left\|\neg \psi^{\prime}\left\{\neg x_{1} / x_{1}, \ldots, \neg x_{n} / x_{n}\right\}\right\|_{\rho}
\end{aligned}
$$

Hence, let $\phi^{\prime} \hat{=} \neg \psi^{\prime}\left\{\neg x_{1} / x_{1}, \ldots, \neg x_{n} / x_{n}\right\}$. It is obvious that no + occurs in $\phi^{\prime}, \phi^{\prime}$ is strictly guarded and strongly positive and $\phi \stackrel{b c}{\Leftrightarrow} \phi^{\prime}$.

. $\phi=\langle A\rangle \phi_{1}$

As $\phi$ is strictly guarded and strongly positive, this implies the following two cases:

1. $\phi_{1}$ is equivalent to a disjunction of some formulae of the form $x_{1} \wedge \cdots x_{n} \wedge$ $\chi_{1} \wedge \cdots \wedge \chi_{\ell}$, where $n, \ell \geq 0, x_{1}, \cdots, x_{n} \in \operatorname{Var}$, and for each $1 \leq i \leq \ell$, $\chi_{i} \in \mathcal{L}_{\mu}($ Act $)$ which is strictly guarded and strongly positive;

2. $\phi_{1}$ is strictly guarded and strongly positive.

In either of the two cases, by the induction hypothesis, it is easy to construct a formula $\phi^{\prime}$ in which no + occurs such that $\phi^{\prime}$ is strictly guarded and $\phi^{\prime} \stackrel{b c}{\Leftrightarrow} \phi$. - $\phi=\phi_{1}+\phi_{2}$

Since $\phi$ is strictly guarded and strongly positive, so are $\phi_{1}$ and $\phi_{2}$. By the induction hypothesis, there exist $\phi_{i}^{\prime}$ such that $\phi_{i}^{\prime}$ is strongly positive and strictly guarded, $\phi_{i}^{\prime} \stackrel{b c}{\Leftrightarrow} \phi_{i}$ and no + occurs in $\phi_{i}^{\prime}$ for $i=1,2$.

We consider the following two cases:

1. $\phi_{1}^{\prime} \stackrel{b c}{\Leftrightarrow} f f$ or $\phi_{2}^{\prime} \stackrel{b c}{\Leftrightarrow} f f$. If so, let $\phi^{\prime} \widehat{=} f f$. By Lemma 5.(1), we have that $\phi_{1}^{\prime}+\phi_{2}^{\prime} \stackrel{b c}{\Leftrightarrow} f f$. On the other hand, by Proposition 1, it follows that $\phi \stackrel{b c}{\Leftrightarrow} f f$. Hence, $\phi^{\prime}$ is what we want.

2. $\phi_{1}^{\prime} \stackrel{b c}{\Leftrightarrow} f f$ and $\phi_{2}^{\prime} \nLeftarrow$ 占 $f f$. Using the laws of Boolean Algebra, Lemma 5.9-12 and Lemma 6 , we can transform $\phi_{1}^{\prime}$ and $\phi_{2}^{\prime}$ equivalently as follows:

$$
\begin{aligned}
\phi_{1}^{\prime} & \Leftrightarrow \bigvee_{i=1}^{m_{1}}\left(\bigwedge_{j=1}^{m_{1, i}} \bigwedge_{h=1}^{m_{1, i, j}}\left\langle A_{1, i, j}\right\rangle \phi_{1, i, j, h} \wedge \bigwedge_{j=1}^{m_{1, i}^{\prime}}\left[B_{1, i, j}\right] \psi_{1, i, j}\right), \\
\phi_{2}^{\prime} & \Leftrightarrow \bigvee_{i=1}^{m_{2}}\left(\bigwedge_{j=1}^{m_{2, i}} \bigwedge_{h=1}^{m_{2, i, j}}\left\langle A_{2, i, j}\right\rangle \phi_{2, i, j, h} \wedge \bigwedge_{j=1}^{m_{2, i}^{\prime}}\left[B_{2, i, j}\right] \psi_{2, i, j}\right),
\end{aligned}
$$

where

- $\forall 1 \leq i \leq 2, \forall 1 \leq j \leq m_{i} \cdot\left(\forall 1 \leq k_{1}, k_{2} \leq m_{i, j} \cdot k_{1} \neq k_{2} \Rightarrow A_{i, j, k_{1}} \cap\right.$ $\left.A_{i, j, k_{2}}=\emptyset\right) \wedge\left(\forall 1 \leq k_{1}, k_{2} \leq m_{i, j}^{\prime} \cdot k_{1} \neq k_{2} \Rightarrow B_{i, j, k_{1}} \cap B_{i, j, k_{2}}=\emptyset\right) \wedge$ $\left(\forall 1 \leq k_{1} \leq m_{i, j}, \forall 1 \leq k_{2} \leq m_{i, j}^{\prime} \cdot A_{i, j, k_{1}} \subseteq B_{i, j, k_{2}} \vee A_{i, j, k_{1}} \cap B_{i, j, k_{2}}=\right.$ $\emptyset))$;

- $B_{1, i_{1}, j_{1}}=B_{2, i_{2}, j_{2}}$ or $B_{1, i_{1}, j_{1}} \cap B_{2, i_{2}, j_{2}}=\emptyset$ for all $1 \leq i_{1} \leq m_{1}, 1 \leq$ $j_{1} \leq m_{1, i_{1}}^{\prime}, 1 \leq i_{2} \leq m_{2}, 1 \leq j_{2} \leq m_{1, i_{2}}^{\prime}$; 
. for all $i=1,2,1 \leq j_{1} \leq m_{i}, 1 \leq k_{1} \leq m_{i, j_{1}} 1 \leq j_{2} \leq m_{3-i}, 1 \leq k_{2} \leq$ $m_{3-i, j_{2}}^{\prime}, A_{i, j_{1}, j_{2}} \subseteq B_{3-i, j_{2}, k_{2}}$ or $A_{i, j_{1}, j_{2}} \cap B_{3-i, j_{2}, k_{2}}=\emptyset$.

By Lemma 5.5-8, we have

$$
\begin{aligned}
& \phi_{1}^{\prime}+\phi_{2}^{\prime} \stackrel{b c}{\Leftrightarrow} \bigvee_{i_{1}=1}^{m_{1}} \bigvee_{i_{2}=1}^{m_{2}}\left(\bigwedge_{j=1}^{m_{1, i_{1}}} \bigwedge_{h=1}^{m_{1, i_{1}, j}}\left\langle A_{1, i_{1}, j}\right\rangle \phi_{1, i_{1}, j, h} \wedge \bigwedge_{j=1}^{m_{1, i_{1}}^{\prime}}\left[B_{1, i_{1}, j}\right] \psi_{1, i_{1}, j}\right)+ \\
&\left(\bigwedge_{j=1}^{m_{2, i_{2}}} \bigwedge_{h=1}^{m_{2, i_{2}, j}}\left\langle A_{2, i_{2}, j}\right\rangle \phi_{2, i_{2}, j, h} \wedge \bigwedge_{j=1}^{m_{2, i_{2}}^{\prime}}\left[B_{2, i_{2}, j}\right] \psi_{2, i_{2}, j}\right)
\end{aligned}
$$

Thus, according to Lemma 5 and Lemma 7, for each disjunct of the right hand of (6), there is a formula $\varphi_{i, j}$ that is equivalent to the disjunct w.r.t. $B C V$, strictly guarded, strongly positive and no + occurs in it, where $1 \leq i \leq m_{1}$ and $1 \leq j \leq m_{2}$. So, let $\phi^{\prime} \widehat{=} \bigvee_{i=1}^{m_{1}} \bigvee_{j=1}^{m_{2}} \varphi_{i, j}$. It is easy to see that $\phi^{\prime}$ meets the requirement.

$\phi=\mu x . \phi_{1}$

Since $\phi$ is strictly guarded and strongly positive, so is $\phi_{1}$. Therefore, by the induction hypothesis, there exists $\phi_{1}^{\prime}$ in which no + occurs such that $\phi_{1}^{\prime}$ is strictly guarded and strongly positive and $\phi_{1}^{\prime} \stackrel{b c}{\Leftrightarrow} \phi_{1}$. By Lemma 4 , it is easy to see that $\mu x . \phi_{1} \stackrel{b c}{\Leftrightarrow} \mu x . \phi_{1}^{\prime}$. Thus, let $\phi^{\prime} \widehat{=} \mu x . \phi_{1}^{\prime}$.

Finally, in order to encode $c \mathcal{L}_{\mu}^{+}(A c t)$ into $c \mathcal{L}_{\mu}(A c t)$, we need to show the following lemma:

Lemma 9. For any $\phi \in c \mathcal{L}_{\mu}^{+}($Act $)$, there exists $\phi^{\prime} \in c \mathcal{L}_{\mu}^{+}($Act $)$such that $\phi^{\prime}$ is strictly guarded and $\phi \Leftrightarrow \phi^{\prime}$.

Proof. In order to prove the lemma, we need to show the following equations:

$$
\begin{aligned}
\mu x . \phi_{1}\left[\langle A\rangle \phi_{2}\left[\left(x \odot \phi_{3}\right)+\phi_{4}\right]\right] & \Leftrightarrow \mu x . \phi_{1}\left[\langle A\rangle \phi_{2}\left[\mu y \cdot\left(\phi_{1}\left[\langle A\rangle \phi_{2}[y]\right] \odot \phi_{3}\right)+\phi_{4}\right]\right] \\
\nu x . \phi_{1}\left[\langle A\rangle \phi_{2}\left[\left(x \odot \phi_{3}\right)+\phi_{4}\right]\right] & \Leftrightarrow \nu x . \phi_{1}\left[\langle A\rangle \phi_{2}\left[\nu y \cdot\left(\phi_{1}\left[\langle A\rangle \phi_{2}[y]\right] \odot \phi_{3}\right)+\phi_{4}\right]\right] \\
\mu x . \phi_{1}\left[[A] \phi_{2}\left[\left(x \odot \phi_{3}\right)+\phi_{4}\right]\right] & \Leftrightarrow \mu x . \phi_{1}\left[[A] \phi_{2}\left[\mu y \cdot\left(\phi_{1}\left[[A] \phi_{2}[y]\right] \odot \phi_{3}\right)+\phi_{4}\right]\right] \\
\nu x . \phi_{1}\left[[A] \phi_{2}\left[\left(x \odot \phi_{3}\right)+\phi_{4}\right]\right] & \Leftrightarrow \nu x . \phi_{1}\left[[A] \phi_{2}\left[\nu y \cdot\left(\phi_{1}\left[[A] \phi_{2}[y]\right] \odot \phi_{3}\right)+\phi_{4}\right]\right] \\
\mu x . \phi_{1}\left[\langle A\rangle \phi_{2}\left[\left(\neg x \odot \phi_{3}\right)+\phi_{4}\right]\right] & \Leftrightarrow \mu x . \phi_{1}\left[\langle A\rangle \phi_{2}\left[\nu y \cdot\left(\neg \phi_{1}\left[\langle A\rangle \phi_{2}[y]\right] \odot \phi_{3}\right)+\phi_{4}\right]\right] \\
\nu x . \phi_{1}\left[\langle A\rangle \phi_{2}\left[\left(\neg x \odot \phi_{3}\right)+\phi_{4}\right]\right] & \Leftrightarrow \nu x . \phi_{1}\left[\langle A\rangle \phi_{2}\left[\mu y \cdot\left(\neg \phi_{1}\left[\langle A\rangle \phi_{2}[y]\right] \odot \phi_{3}\right)+\phi_{4}\right]\right] \\
\mu x . \phi_{1}\left[[A] \phi_{2}\left[\left(\neg x \odot \phi_{3}\right)+\phi_{4}\right]\right] & \Leftrightarrow \mu x . \phi_{1}\left[[A] \phi_{2}\left[\nu y \cdot\left(\neg \phi_{1}\left[[A] \phi_{2}[y]\right] \odot \phi_{3}\right)+\phi_{4}\right]\right] \\
\nu x . \phi_{1}\left[[A] \phi_{2}\left[\left(\neg x \odot \phi_{3}\right)+\phi_{4}\right]\right] & \Leftrightarrow \nu x . \phi_{1}\left[[A] \phi_{2}\left[\mu y \cdot\left(\neg \phi_{1}\left[[A] \phi_{2}[y]\right] \odot \phi_{3}\right)+\phi_{4}\right]\right]
\end{aligned}
$$

where $\odot \in\{\wedge, \vee\}, \phi_{i}[]$ stands for a formula with the hole [ ], the formula at the left side of each equation is guarded.

We only prove (9) as an example, the others can be proved similarly. ${ }^{1}$

Since $\phi_{1}\left[[A] \phi_{2}\left[\left(x \odot \phi_{3}\right)+\phi_{4}\right]\right]$ is guarded, by Knaster-Tarski Theorem, it is clear that $\mu x . \phi_{1}\left[[A] \phi_{2}\left[\left(x \odot \phi_{3}\right)+\phi_{4}\right]\right]$ is the unique least solution of the equation

$$
x=\phi_{1}\left[[A] \phi_{2}\left[\left(x \odot \phi_{3}\right)+\phi_{4}\right]\right]
$$

${ }^{1}$ Note that in the proofs for $(15)-(14)$, we need to let $\neg y=\left(\neg x \odot \phi_{3}\right)+\phi_{4}$ in order to guarantee the resulted formulae are still positive. 
Let $y$ be a fresh variable and $y=\left(x \odot \phi_{3}\right)+\phi_{4}$. It is easy to see the least solution of (15) is equivalent to the $x$-component of the least solution of the following equation system:

$$
\begin{aligned}
& x=\phi_{1}\left[[A] \phi_{2}\left[\left(x \odot \phi_{3}\right)+\phi_{4}\right]\right] \\
& y=\left(x \odot \phi_{3}\right)+\phi_{4}
\end{aligned}
$$

Meanwhile, it is easy to rewrite the above equation system to the following one

$$
\begin{aligned}
x & =\phi_{1}\left[[A] \phi_{2}[y]\right] \\
y & =\left(\phi_{1}\left[[A] \phi_{2}[y]\right] \odot \phi_{3}\right)+\phi_{4}
\end{aligned}
$$

It is not hard to derive the least solution of the above equation system as

$$
\left(\mu x . \phi_{1}\left[[A] \phi_{2}\left[\mu y \cdot\left(\phi_{1}\left[[A] \phi_{2}[y]\right] \odot \phi_{3}\right)+\phi_{4}\right]\right], \mu y \cdot\left(\phi_{1}\left[[A] \phi_{2}[y]\right] \odot \phi_{3}\right)+\phi_{4} .\right.
$$

Therefore, (9) follows.

Repeatedly applying (7)-(14), for any given formula $\phi \in c \mathcal{L}_{\mu}^{+}($Act $)$, we can rewrite it to $\phi^{\prime}$ which is strictly guarded such that $\phi \Leftrightarrow \phi^{\prime}$.

Example 1. Let $\phi=\mu x .\langle A\rangle x+\mu y .[C] \neg(\langle B\rangle \neg y+\neg x) \vee\langle C\rangle t t$, where $A \cap B=$ $B \cap C=A \cap C=\emptyset$. Applying the rewriting rule (13), it results that

$$
\begin{aligned}
\phi \Leftrightarrow & \mu x \cdot\langle A\rangle x+ \\
& \mu y \cdot\left([C] \neg\left(\nu z . \neg\left(\langle A\rangle x+\mu y^{\prime} \cdot([C] \neg z \vee\langle C\rangle t t)\right)+\langle B\rangle \neg y\right) \vee\langle C\rangle t t\right) \\
\Leftrightarrow & \mu x \cdot\langle A\rangle x+\mu y \cdot([C] \neg(\nu z . \neg(\langle A\rangle x+([C] \neg z \vee\langle C\rangle t t))+\langle B\rangle \neg y) \vee\langle C\rangle t t) \\
\Leftrightarrow & \mu x \cdot\langle A\rangle x+\mu y \cdot([C] \neg(\nu z . \neg\langle A\rangle x+\langle B\rangle \neg y) \vee\langle C\rangle t t) \\
& \Leftrightarrow \mu x \cdot\langle A\rangle x+\mu y \cdot([C] \neg(\neg\langle A\rangle x+\langle B\rangle \neg y) \vee\langle C\rangle t t) \\
& \Leftrightarrow \mu x .\langle A\rangle x+\mu y \cdot([C][B] y \vee\langle C\rangle t t) \\
& \Leftrightarrow \mu x \cdot\langle A\rangle x \vee(\langle A\rangle x \wedge\langle C\rangle t t) \\
& \Leftrightarrow \mu x .\langle A\rangle x,
\end{aligned}
$$

where $\phi_{1}=\langle A\rangle x+\mu y \cdot([] \vee\langle C\rangle t t), \phi_{2}=\neg([]), \phi_{3}=t t, \phi_{4}=\langle B\rangle \neg y$.

Note that in the above example, we can also unfold $\mu y \cdot[C] \neg(\langle B\rangle \neg y+\neg x) \vee$ $\langle C\rangle t t$ first, then apply Lemma 7 and obtain the same result.

Directly from Lemma 9 and Lemma 8, we can conclude:

Theorem 2. $\forall \phi \in c \mathcal{L}_{\mu}^{+}(A c t), \exists \phi^{\prime} \in c \mathcal{L}_{\mu}($ Act $) . \phi \Leftrightarrow \phi^{\prime}$.

In the later, we will use $E n$ to denote the above implicit translating function from $c \mathcal{L}_{\mu}^{+}(A c t)$ to $c \mathcal{L}_{\mu}(A c t)$.

\section{Synchronization Tree Logic}

[2] proposed a logic, called Synchronization Tree Logic (STL) for the specification and proof of programs, described by $\mathcal{T}[\{\epsilon\},\{+\}$, Act, $\mathcal{X}]$. Formulae of STL can 
be obtained from the constants $\epsilon, \top$ by using logical connectives, consistent extensions of the operators $a \in$ Act, + and fixpoint operators. Therefore, STL contains $\mathcal{T}[\{\epsilon\},\{+\}, A c t, \mathcal{X}]$, i.e., terms of $\mathcal{T}[\{\epsilon\},\{+\}, A c t, \mathcal{X}]$ are formulae of STL if we look recursive operators of $\mathcal{T}[\{\epsilon\},\{+\}, A c t, \mathcal{X}]$ as greatest fixpoint operators. Its semantics is defined by associating with a formula a set of terms (synchronization trees) representing unions of congruence classes of the strong congruence relation.

Given a set $A c t$ of atomic actions and a set $\mathcal{X}$ of variables, formulae of STL are constructed by the rule:

$$
\phi::=\epsilon|\top| x|\neg \phi| B \phi\left|\phi+\phi^{\prime}\right| \phi \vee \phi^{\prime} \mid \mu x . \phi
$$

where $x \in \mathcal{X}$ and $B \subseteq$ Act.

In what follows, we will use $\mathcal{L}_{\mathrm{STL}}(A c t)$ to stand for the set of formulae of STL that are guarded and positive and $c \mathcal{L}_{\mathrm{STL}}(A c t)$ for the subset of $\mathcal{L}_{\mathrm{STL}}(A c t)$ in which all formulae are closed.

Definition 6. Given a valuation $\rho \in B C V$, the semantics of $\mathcal{L}_{\mathrm{STL}}($ Act $)$ is given by a satisfaction relation between $\mathcal{T}[\{\epsilon\},\{+\}$, Act, $\mathcal{X}]$ and $\mathcal{L}_{\mathrm{STL}}($ Act $)$ relative to $\rho$, denoted by $\models_{\mathrm{STL}}^{\rho}$, inductively defined as follows:

$$
\begin{gathered}
P \models_{\mathrm{STL}}^{\rho} \top, \\
P \models_{\mathrm{STL}}^{\rho} \epsilon \text { iff } P \sim \epsilon, \\
P \models_{\mathrm{STL}}^{\rho} \neg \phi \text { iff } P \models_{\mathrm{STL}}^{\rho} \phi, \\
P \models_{\mathrm{STL}}^{\rho} B \phi \text { iff } \exists I \subseteq \mathbb{N} . I \neq \emptyset, I \text { is finite, } \\
\forall i \in I\left(\exists a_{i} \in B \text { and } \exists P_{i} . P_{i} \models_{\mathrm{STL}}^{\rho} \phi\right), P \sim \Sigma_{i \in I} a_{i} P_{i}, \\
P \models_{\mathrm{STL}}^{\rho} \phi_{1} \vee \phi_{2} \text { iff } P \models_{\mathrm{STL}}^{\rho} \phi_{1} \text { or } P \models_{\mathrm{STL}}^{\rho} \phi_{2}, \\
P \models{ }_{\mathrm{STL}}^{\rho} \phi_{1}+\phi_{2} \text { iff } \exists P_{1}, P_{2} . P_{1}=_{\mathrm{STL}}^{\rho} \phi_{1}, P_{2} \models_{\mathrm{STL}}^{\rho} \phi_{2} \text { and } P \sim P_{1}+P_{2}, \\
P \models_{\mathrm{STL}}^{\rho} \mu x . \phi \text { iff } P \in \bigcap\left\{\mathcal{A} \mid \mathcal{A} \text { is B.C. and }\|\phi\|_{\rho[x \sim \mathcal{A}]} \subseteq \mathcal{A}\right\},
\end{gathered}
$$

where $\mathcal{A} \subseteq \mathcal{T}[\{\epsilon\},\{+\}, A c t, \mathcal{X}], B \subseteq$ Act.

Some notions and derived operators can be defined similarly as in $\mu \mathrm{M}$ and $\mu \mathrm{M}^{+}$. In what follows we will use $\perp$ to denote $\neg \top$. Note that in STL all valuations are restricted to be in $B C V$.

[2] proved the following results:

Proposition 4. $\|\phi\|_{\rho}$ is B.C., for any $\rho \in B C V$ and $\phi \in \mathcal{L}_{\mathrm{STL}}($ Act $)$.

Proposition 5. For each $P \in \mathcal{T}[\{\epsilon\},\{+\}$, Act, $\mathcal{X}]$,

$$
\left\|\phi_{P}\right\|=\left\{P^{\prime} \in \mathcal{T}[\{\epsilon\},\{+\}, \text { Act, } \mathcal{X}] \mid P \sim P^{\prime}\right\}
$$

More results on STL can be found in [2]. 


\section{Reducing $c \mathcal{L}_{\mathrm{STL}}($ Act $)$ to $c \mathcal{L}_{\mu}($ Act $)$}

In this section, we define a function $\operatorname{Tr}: \mathcal{L}_{\mathrm{STL}}(A c t) \rightarrow \mathcal{L}_{\mu}^{+}(A c t)$ such that for any $\phi \in \mathcal{L}_{\mathrm{STL}}(A c t), P \in \mathcal{T}[\{\epsilon\},\{+\}, A c t, \mathcal{X}]$ and $\rho \in B C V, P \models_{\mathrm{STL}}^{\rho} \phi$ iff $P \models{ }_{\mu \mathrm{M}^{+}}^{\rho} \operatorname{Tr}(\phi)$. Moreover, according to Theorem 2, for each $\phi \in c \mathcal{L}_{\mathrm{STL}}(A c t)$ and $P \in \mathcal{T}[\{\epsilon\},\{+\}, A c t, \mathcal{X}], P={ }_{\mu \mathrm{M}^{+}} \operatorname{Tr}(\phi)$ iff $P \mid{ }_{\mu \mathrm{M}} \operatorname{En}(\operatorname{Tr}(\phi))$. Thus, this completes the reduction from $c \mathcal{L}_{\mathrm{STL}}(A c t)$ to $c \mathcal{L}_{\mu}(A c t)$.

Definition 7. The function $\operatorname{Tr}$ is inductively defined as follows: $\operatorname{Tr}(\perp) \hat{=} f f$, $\operatorname{Tr}(\top) \hat{=} t t, \operatorname{Tr}(x) \hat{=} x, \operatorname{Tr}(\epsilon) \hat{=}[A c t] f f, \operatorname{Tr}(\neg \phi) \hat{=} \neg \operatorname{Tr}(\phi), \operatorname{Tr}(B \phi) \hat{=}[B] \operatorname{Tr}(\phi) \wedge$ $[\bar{B}] f f \wedge\langle B\rangle \operatorname{Tr}(\phi), \operatorname{Tr}\left(\phi_{1} \vee \phi_{2}\right) \hat{=} \operatorname{Tr}\left(\phi_{1}\right) \vee \operatorname{Tr}\left(\phi_{2}\right), \operatorname{Tr}\left(\phi_{1}+\phi_{2}\right) \bumpeq \widehat{T} \operatorname{Tr}\left(\phi_{1}\right)+\operatorname{Tr}\left(\phi_{2}\right)$, $\operatorname{Tr}(\mu x . \phi) \hat{=} \mu x \cdot \operatorname{Tr}(\phi)$.

Theorem 3. For any $P \in \mathcal{T}[\{\epsilon\},\{+\}, A c t, \mathcal{X}]$ and $\phi \in \mathcal{L}_{\mathrm{STL}}($ Act $), \operatorname{Tr}(\phi) \in$ $\mathcal{L}_{\mu}^{+}($Act $)$and $P \models_{\text {STL }}^{\rho} \phi$ iff $P \models_{\mu \mathrm{M}^{+}}^{\rho} \operatorname{Tr}(\phi)$. Where $\rho \in B C V$.

Proof. $\operatorname{Tr}(\phi) \in \mathcal{L}_{\mu}^{+}(A c t)$ is obvious by Definition 7 , the proof for the second part can proceed by induction on the structure of $\phi$.

The following theorem that follows directly from Theorem 3 and Theorem 2 indicates that applying $T r$ and $E n$, STL can be translated into $\mu \mathrm{M}$.

Theorem 4. For all $P \in \mathcal{T}[\{\epsilon\},\{+\}, A c t, \mathcal{X}], \phi \in c \mathcal{L}_{\mathrm{STL}}(A c t), \operatorname{En}(\operatorname{Tr}(\phi)) \in$ $c \mathcal{L}_{\mu}($ Act $)$ and $P \models_{\mathrm{STL}} \phi$ iff $P \models_{\mu \mathrm{M}} \operatorname{En}(\operatorname{Tr}(\phi))$.

Corollary 1. For any $P, Q \in \mathcal{T}[\{\epsilon\},\{+\}, A c t, \mathcal{X}], Q \models_{\mu \mathrm{M}} \operatorname{En}\left(\operatorname{Tr}\left(\phi_{P}\right)\right)$ iff $P \sim$ $Q$.

Below we present an example to show how to translate a formula $\phi \in$ $c \mathcal{L}_{\mathrm{STL}}(A c t)$ into $c \mathcal{L}_{\mu}(A c t)$, and indicate that for any $P \in \mathcal{T}[\{\epsilon\},\{+\}, A c t, \mathcal{X}]$, $\operatorname{En}\left(\operatorname{Tr}\left(\phi_{P}\right)\right)$ is exactly the characteristic formula of $P$ up to $\sim$. Given an equivalence or preorder $\preceq$ over processes, the characteristic formula for a process $P$ up to it is a formula $\phi_{P}$ such that given a process $Q, Q \models \phi_{P}$ if and only if $Q \preceq P$. Example 2. Suppose Act $=\{a, b, c\}, P \widehat{=} r e c x .(a b x+a c \epsilon)$ and $Q \widehat{=} r e c x .[a(b x+$ $c \epsilon)]$. Thus, by Definition 7,

$$
\begin{aligned}
\operatorname{Tr}\left(\phi_{P}\right) \Leftrightarrow \nu x & {[\langle a\rangle(\langle b\rangle x \wedge[\{\bar{b}\}] f f \wedge[b] x) \wedge[\{\bar{a}\}] f f \wedge[a](\langle b\rangle x \wedge[\{\bar{b}\}] f f \wedge[b] x)] } \\
+ & {[\langle a\rangle(\langle c\rangle[\text { Act }] f f \wedge[\{\bar{c}\}] f f \wedge[c][\text { Act }] f f) \wedge[\{\bar{a}\}] f f \wedge[a](\langle c\rangle[\text { Act }] f f} \\
& \wedge[\{\bar{c}\}] f f \wedge[c][\text { Act }] f f)]
\end{aligned}
$$

Moreover, we can get

$$
\begin{aligned}
\operatorname{En}\left(\operatorname{Tr}\left(\phi_{P}\right)\right) \Leftrightarrow \nu x & .\langle a\rangle(\langle b\rangle x \wedge[\{\bar{b}\}] f f \wedge[b] x) \wedge\langle a\rangle(\langle c\rangle[\text { Act }] f f \wedge[\{\bar{c}\}] f f \\
& \wedge[c][A c t] f f) \wedge[\{\bar{a}\}] f f \wedge[a]((\langle b\rangle x \wedge[\{\bar{b}\}] f f \wedge[b] x) \\
& \vee(\langle c\rangle[\text { Act }] f f \wedge[\{\bar{c}\}] f f \wedge[c][\text { Act }] f f))
\end{aligned}
$$

It is easy to see that $\operatorname{En}\left(\operatorname{Tr}\left(\phi_{P}\right)\right)$ is exactly the characteristic formula of $P$ and $Q \forall_{\mu \mathrm{M}} \operatorname{En}\left(\operatorname{Tr}\left(\phi_{P}\right)\right)$ since $P \nsim Q$. 


\section{Concluding Remarks}

In this paper, we investigated the definability of the non-deterministic operator + introduced in STL as a primitive in the $\mu$-calculus. This was captured via extending the $\mu$-calculus with the non-deterministic operator + to $\mu \mathrm{M}^{+}$first and then showing that $\mu \mathrm{M}^{+}$can be encoded into the modal $\mu$-calculus.

Furthermore, we proved that STL can be translated into the modal $\mu$-calculus by encoding it into $\mu \mathrm{M}^{+}$. Thus, if $A c t$ is finite, we can get the decidability of STL by the decidability of the $\mu$-calculus [5]. In fact, we could translate other STL-like modal logics into the $\mu$-calculus, for example, it is easy to encode the modal process logic presented in [6] into the $\mu$-calculus according to the results shown in this paper.

The converse procedure to translate $\mathcal{L}_{\mu}(A c t)$ into $\mathcal{L}_{\mathrm{STL}}(A c t)$ can be obtained easily. Thus, we see that the $\mu$-calculus is as expressive as STL.

In summary, the significance of this work lies in:

- We proved that the non-deterministic choice + is definable in the $\mu$-calculus, so that we can compare the expressiveness between the $\mu$-calculus with process algebra-like modal logics such as STL, for example, it was shown in this paper that the $\mu$-calculus is as expressive as STL.

- A connection between the connectives of the $\mu$-calculus and the operators of $\mathcal{T}[\{\epsilon\},\{+\}, A c t, \mathcal{X}]$ has been established in this paper. This thus makes it possible that syntax-directed proofs for programs defined in terms of $\mathcal{T}[\{\epsilon\},\{+\}, A c t, \mathcal{X}]$ can be done in the $\mu$-calculus;

- We indirectly presented an algorithm to construct the characteristic formula up to $\sim$ for a given finite-state process specified by $\mathcal{T}[\{\epsilon\},\{+\}, A c t, \mathcal{X}]$ syntactically and compositionally.

\section{References}

1. L. Aceto and M. Hennessy. Termination, deadlock, and divergence. Journal of ACM, Vol. 39, No.1:14\%-18\%. January, 1992.

2. S. Graf and J. Sifakis. A logic for the description of non-deterministic programs and their properties. Information and Control, 68:254-270. 1986.

3. C.A.R. Hoare. Communicating Sequential Processes. Prentice-Hall, 1985.

4. M. Hennessy and R. Milner. Algebraic laws for nondeterminism and concurrency. Journal of ACM, 32:137-161. Jan., 1985.

5. D. Kozen. Results on the propositional mu-calculus. Theoretical Computer Science, 27:333-354. 1983.

6. K.G. Larsen and B. Thomsen. A modal process logic. In the proc. of LICS'88, pp.203-210. IEEE Computer Science Society, 1988.

7. R. Milner. A complete inference system for a class of regular behaviours. Journal of Computer and System Sciences, 28:439-466. 1984.

8. R. Milner. Communication and Concurrency. Prentice Hall, 1989.

9. C. Stirling. Modal and Temporal Properties of Processes. Springer-Verlag, 2001.

10. A. Tarski. A lattice-theoretical fixpoint theorem and its application. Pacific J. Math., 5:285-309. 1955.

11. I. Walukiewicz. Completeness of Kozen's axiomatisation of the propositional Mucalculus. Information and Computation, 157:142-182. 2000. 